06

\title{
Каскадная генерация второй гармоники с половинным порядком периодической ориентации
}

\author{
() В.Ю. Мыльников, Н.С. Аверкиев, Г.С. Соколовский \\ Физико-технический институт им. А.Ф. Иофрфе РАН, \\ 194021 Санкт-Петербург, Россия \\ ฯ e-mail: gs@mail.ioffe.ru \\ Поступила в редакцию 28.06.2020 г. \\ В окончательной редакции 28.06.2020 г. \\ Принята к публикации 16.07.2020 г.
}

\begin{abstract}
Теоретически продемонстрирована каскадная генерация второй и четвертой гармоник в периодически поляризованном нелинейном кристалле с половинным порядком периодической ориентации. Рассмотрен каскадный процесс, в котором четыре фотона фундаментальной гармоники сначала преобразуются в промежуточный фотон четвертой гармоники, который на втором этапе параметрически распадается на два фотона второй гармоники. Показано, что можно добиться дробного фазового синхронизма при помощи использования несимметричной решетки периодической поляризации. Для описания распространения и преобразования света внутри нелинейного кристалла использованы квантовые пространственные уравнения Гейзенберга, с помощью которых были рассчитаны средние числа фотонов четвертой и второй гармоник как функции длины нелинейного кристалла.
\end{abstract}

Ключевые слова: генерация второй гармоники, нелинейная оптика, фазовый синхронизм.

DOI: $10.21883 /$ OS.2020.11.50175.199-20

\section{Введение}

Создание лазера с широким диапазоном перестройки, излучающего в видимом диапазоне, мотивированно запросами биомедицинского сообщества, биофотоники, флуоресцентной микроскопии и спектроскопии. Однако из-за того, что перестройка, как правило, достигается за счет использования нескольких источников излучения, такие системы громоздки, дороги и сложны для изготовления, настройки и эксплуатации. Использование эффекта генерации второй гармоники (ГВГ) позволяет избежать эти сложности и представляется многообещающим способом достижения перестраиваемой генерации излучения видимого диапазона. Лазерный источник, основанный на инфракрасном полупроводниковом лазере и удвоении частоты в нелинейном кристалле [1-3], в перспективе должен стать заменой газовых и твердотельных лазеров, используемых в данный момент для широкого класса биомедицинских приложений.

Из-за дисперсии показателя преломления в нелинейном кристалле в общем случае не выполняется закон сохранения импульса и эффективная ГВГ происходит только на масштабе так называемой длины когерентности: $L_{c}=1 / 2 \lambda /|n(\lambda)-n(2 \lambda)|[4]$, где $\lambda-$ длина волны 2-й гармоники, а $n(\lambda)$ и $n(2 \lambda)$ - коэффициенты преломления кристалла на длине волны 2-й (ВГ) и фундаментальной гармоник (ФГ) соответственно. В настоящий момент наиболее используемым методом преодоления проблемы нарушения закона сохранения импульса или „синхронизации фаз“ является периодическая ориентация нелинейного кристалла под действием высокого напряжения с периодом, равным двум длинам когерентности $\Lambda=2 L_{c}$. В результате, рассогласование импульсов для ГВГ $k(\lambda)-2 k(2 \lambda)$ в точности компенсируется волновым вектора решетки периодической ориентации $q_{0}=2 \pi / \lambda$. В работе Фейера и др. [5] было показано, что рассогласование фаз может быть скомпенсировано не одним, а несколькими волновыми векторами решетки так, что условие фазового синхронизма будет принимать вид

$$
k(\lambda)-2 k(2 \lambda)=s q_{0},
$$

где $s=1,2,3$ и т.д. При этом увеличение $s$ приводит к сдвигу частоты ГВГ в синюю область спектра. В настоящей работе мы будем обсуждать, в каких физических процессах число $s$ может принимать дробное значение, что для $s<1$ приводит к красному сдвигу ГВГ [6]. Далее нами будет показано, что $s$ может принимать дробное значение. Данный режим реализуется в том случае, если предположить, что в процессе ГВГ участвует $p$ пар фотонов ФГ, $p$ фотонов ВГ и $r$ векторов решетки. В результате, условие фазового синхронизма изменяется следующим образом [7]:

$$
k(\lambda)=2 k(2 \lambda)=r / p q_{0} .
$$

В рамках данной работы теоретически проанализируем физический процесс, в рамках которого две пары фотонов ФГ $(p=2)$ эффективно взаимодействуют с одним волновым вектором решетки $(r=1)$, превращаясь в два фотона ВГ, что соответствует половинному порядку фазового синхронизма $(s=1 / 2)$. 

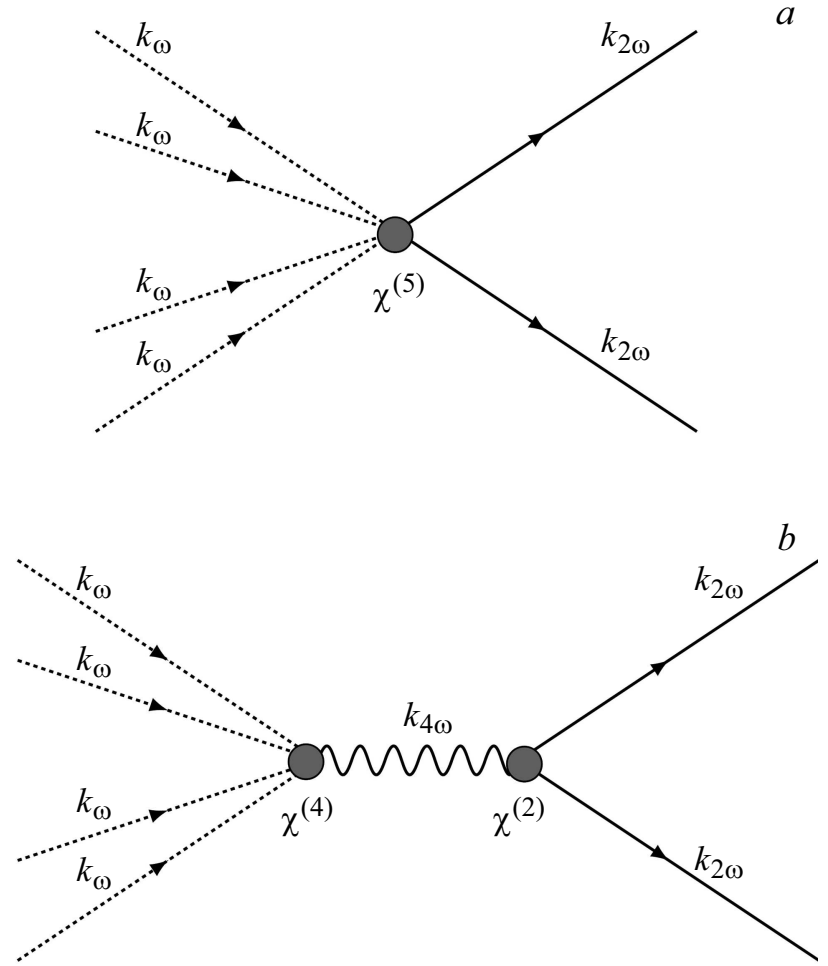

Рис. 1. (a) Прямой процесс преобразования 4 фотонов ФГ в 2 фотона ВГ за счет нелинейности $\chi^{(5)}$. (b) Каскадный процесс, в котором на первом этапе 4 фотона ФГ рождают фотон четвертой гармоники за счет нелинейности $\chi^{(4)}$, а затем промежуточный фотон распадается на 2 фотона ВГ за счет нелинейности $\chi^{(2)}$.

\section{Теория}

Теоретическое исследование механизмов согласования фаз ГВГ при дробном порядке периодической ориентации нелинейного кристалла следует начать с обсуждения нелинейных процессов, для которых такая генерация осуществима. Как обсуждалось выше, для полуцелого фазового синхронизма вместо двух фотонов ФГ и одного фотона ВГ мы будем рассматривать процесс, в котором участвуют 4 фотона ФГ и 2 фотона ВГ, как показано на рис. $1, a$ и $b$.

В случае прямого процесса (рис. 1,a) 4 фотона ФГ напрямую преобразуются в 2 фотона ВГ за счет нелинейности $\chi^{(5)}$. Однако так как $\chi^{(5)}$ является компонентой тензора 6-го ранга, то при совершении операции инверсии оси $z \rightarrow-z$, данный тензор не будет менять знак. Из-за этого $\chi^{(5)}$ в периодически-ориентированном кристалле не будет зависеть от координаты $z$ и не сможет обеспечить выполнения фазового синхронизма волновых векторов.

Более интересная ситуация возникает, если рассмотреть каскадный процесс (рис. 1, $b$ ), в котором четыре фотона ВГ сначала преобразовываются в промежуточный фотон 4-й гармоники (ЧГ), который на втором этапе параметрически распадается на 2 фотона ВГ.
Нелинейности $\chi^{(2)}$ и $\chi^{(4)}$ в отличие от $\chi^{(5)}$ являются компонентами тензора нечетного третьего и пятого ранга соответственно. В периодически-ориентированном кристалле данные нелинейности будут менять знак, за счет чего возможно обеспечить дробный порядок фазового синхронизма. В настоящей работе мы будем предполагать, что нелинейный процесс осуществляется за счет компонент тензоров $\chi_{z z z}^{(5)}$ и $\chi_{z z z z z}^{(5)}$, в связи с чем все волны для рассматриваемого процесса будут необыкновенными и поляризационная зависимость будет определяться величиной проекции вектора электрического поля фундаментальной гармоники $E$ на ось $z$.

Распространение и нелинейное взаимодействие света в кристалле принято описывать при помощи операторных уравнений Гейзенберга, зависящих от пространственной координаты $x$, которые определяют эволюцию электромагнитного поля внутри нелинейного кристалла $[8,9]$. Наличие дисперсии показателя преломления позволяет ограничиться взаимодействием всего трех резонансных мод электромагнитного поля. Следуя логике, развитой в работе [8], запишем оператор плотности импульса $G$ в виде суммы двух слагаемых: $G=G_{P 0}+G_{N L}$, где $G_{0}$ описывает наличие дисперсии показателя преломления нелинейного кристалла:

$$
\hat{G}_{0}=\hbar k_{\omega} \hat{e}^{\dagger} \hat{e}+\hbar k_{2 \omega} \hat{a}+\hbar k_{4 \omega} \hat{b}^{\dagger} \hat{b}
$$

В выражении (1) $\hbar$ - постоянная Планка, $\hat{e}-$ оператор уничтожения моды с частотой $\omega, \hat{a}-$ с частотой $2 \omega$, $\hat{b}-$ с частотой $4 \omega$. Все вышеперечисленные операторы удовлетворяют бозонным коммутационным соотношениям. Кроме того, $k_{\omega}=n(\omega) \omega / c-$ волновой вектор света с частотой $\omega$. Зависимость показателя преломления $n(\omega)$ от частоты $\omega$ приводит к нарушению выполнения закона сохранения импульса в кристалле.

В свою очередь, $G_{N L}$ отвечает за нелинейное взаимодействие мод и определяется следующим выражением:

$$
\begin{aligned}
\hat{G}_{N L}= & -i \hbar \kappa^{(4)} g(x)\left(\hat{b}^{\dagger} \hat{e}^{4}-\text { h.c. }\right) \\
& -i \hbar \kappa^{(2)} g(x)\left(\hat{a}^{\dagger 2} \hat{b}-\text { h.c. }\right),
\end{aligned}
$$

где константа нелинейного взаимодействия $\kappa^{(2,4)}$ пропорциональна $\chi^{(2,4)}$ и имеет размерность волнового вектора. Функция $g(x)$ описывает периодическую ориентацию структуры образца. Первая часть выражения (2) отвечает за рождение промежуточного фотона ЧГ из 4 фотонов ФГ, а вторая часть - за последующий параметрический распад промежуточного фотона ЧГ на 2 фотона ВГ. Эволюция операторов поля при распространении внутри нелинейного кристалла дается следующим операторным уравнением Гейзенберга в соответствии c [8]:

$$
\frac{d \hat{A}}{d x}=\frac{i}{\hbar}\left[\hat{A}(x), \hat{G}_{0}+\hat{G}_{N L}\right] .
$$

Используя (3), можно получить операторные уравнения движения для всех операторов электромагнитного 
поля рассматриваемой задачи:

$$
\begin{gathered}
\frac{d \hat{e}(x)}{d x}=i k_{\omega} \hat{e}(x)-4 \kappa^{(4)} g(x) \hat{b}(x) \hat{e}^{\dagger 3}(x), \\
\frac{d \hat{b}(x)}{d x}=i k_{4 \omega} \hat{b}(x)+\kappa^{(4)} g(x) \hat{e}^{4}(x)-\kappa^{(2)} g(x) \hat{a}(x), \\
\frac{d \hat{a}(x)}{d x}=i k_{2 \omega} \hat{a}(x)+2 \kappa^{(2)} g(x) \hat{b}(x) \hat{a}^{\dagger}(x) .
\end{gathered}
$$

Первые слагаемые в правой части уравнений (4) задают для каждого оператора собственный набег фазы, появляющийся за счет распространения света в кристалле. Естественно исключить данные фазовые множители из уравнений движения, перейдя к медленно изменяющимся операторам вида

$$
\begin{gathered}
\hat{e}(x)=\hat{e}_{M}(x) e^{i k_{\omega} x}, \hat{a}(x)=\hat{a}_{M}(x) e^{i k_{2 \omega} x}, \\
\hat{b}(x)=\hat{b}_{M}(x) e^{i k_{4 \omega} x} .
\end{gathered}
$$

Кроме того, будем предполагать поле $\hat{e}_{M}$ классическим и независящим от координаты $x$ (приближение постоянной накачки) и заменим оператор $\hat{e}_{M}$ на его среднее значение $-E_{z}$. После всех преобразований уравнения движения для медленно меняющихся операторов второй и четвертой гармоники примут вид

$$
\begin{gathered}
\frac{d \hat{b}_{M}(x)}{d x}=\kappa^{(4)} g(x) e^{-i \Delta k x} E_{z}^{4}-\kappa^{(2)} g(x) e^{-i(\Delta k-\delta k) x} \hat{a}_{M}(x), \\
\frac{d \hat{a}_{M}(x)}{d x}=2 \kappa^{(2)} g(x) e^{i(\Delta k-\delta k) x} \hat{b}_{M}(x) \hat{a}_{M}^{\dagger}(x) .
\end{gathered}
$$

где были введены следующие обозначения, определяющие величину рассогласования фаз из- за наличия дисперсии показателя преломления:

$$
\begin{gathered}
k_{4 \omega}-4 k_{\omega}=\Delta k, \\
k_{4 \omega}-2 k_{2 \omega}=\Delta k-\delta k, \\
2 k_{2 \omega}-4 k_{\omega}=\delta k .
\end{gathered}
$$

Первая строка в выражении (6) определяет рассогласование процесса генерации промежуточной ЧГ. Вторая строка дает рассогласование процесса распада фотона ЧГ на 2 фотона ВГ. Третья строка - это итоговое рассогласование для процесса превращения исходных 4 фотонов ФГ в 2 фотона ВГ, деление пополам которого дает условие фазового синхронизма ГВГ полуцелого порядка. Как будет показано далее, подбором вида решетки периодической ориентации нелинейного кристалла $g(x)$ возможно получить как синхронизацию только ГВГ, так и всех нелинейных процессов сразу.

Обеспечить фазовый синхронизм для данного нелинейного каскадного процесса становится возможным за счет наличия периодической структуры образца $g(x)$. На рис. 2, $a, b$ показаны примеры решеток с симметричной $(a)$ и несимметричной $(b)$ доменной структурой. Направление периодической ориентации нелинейного кристалла на рис. 2 отображается стрелками. Такак функция $g(x)$ имеет период $\Lambda$, ее можно представить в виде следующего ряда Фурье:

$$
g(x)=\sum_{m=-\infty}^{\infty} g_{m} \exp (\text { iqmx }),
$$

где $q=2 \pi / \Lambda$ - волновой вектор решетки периодической ориентации. Сначала рассмотрим ситуацию, в которой фазовый синхронизм будет осуществлен для всех нелинейных процессов, при одновременном выполнении условия ГВГ в полуцелом порядке. Для этого необходимо подставить формулу (7) в (5) и получить

$$
\begin{gathered}
\frac{d \hat{b}_{M}(x)}{d x}=\kappa^{(4)} \sum_{m=-\infty}^{\infty} g_{m} e^{-i\left(\Delta k-m q_{0}\right) x} E_{0}^{4}, \\
\frac{d \hat{a}_{M}(x)}{d x}=2 \kappa^{(2)} \sum_{\ell=-\infty}^{\infty} g_{\ell}^{*} e^{i\left(\Delta k-\delta k-\ell q_{0}\right) x} \hat{b}_{M}(x) \hat{a}_{M}^{\dagger}(x) .
\end{gathered}
$$

Заметим, что в (8) мы пренебрегли самым правым членом в уравнении движения для ЧГ, считая малым влияние ВГ на поле ЧГ. В итоге из (8) автоматически следует, что фазовый синхронизм всех нелинейных процессов можно обеспечить за счет выполнения следующих двух условий:

$$
\begin{gathered}
\delta k=(m-\ell) q_{0}, \\
\Delta k=m q_{0},
\end{gathered}
$$

где $m$ и $\ell-$ целые положительные числа. Из первого условия (9) следует, что для произвольной частоты ФГ $\omega$ и для соответствующего данной частоте рассогласования $\delta k$ всегда можно подобрать период решетки периодической ориентации $\Lambda=\Lambda_{0}$ и соответствующий ей вектор решетки $q=q_{0}$, чтобы добиться фазового синхронизма для процесса ГВГ. Однако одновременная эффективная генерация промежуточной ЧГ возможна только для определенных частот света $\omega$, которые задаются вторым фазовым условием из (9). Итоговое рассогласование фаз для ГВГ через волновые векторы ФГ и ВГ, используя (9) и (6), можно представить в виде

$$
k_{2 \omega}-2 k_{\omega}=-\frac{(m-\ell)}{2} q_{0}
$$

где величина $(m-\ell) / 2$ является либо целым, либо полуцелым положительным числом. Везде далее мы будем рассматривать случай фазового синхронизма половинного порядка, для которого необходимо, чтобы $\ell=m-1$. Из данного условия следует, что сразу две соседние фурье-компоненты $m$ и $m-1$ решетки $g_{m}$ не должны быть равны нулю. Выполнение фазового синхронизма за счет соседних фурье-компонент дает как возможность получить одновременную генерацию ЧГ и ВГ, так и условие половинного фазового согласования (10) только для ГВГ. 

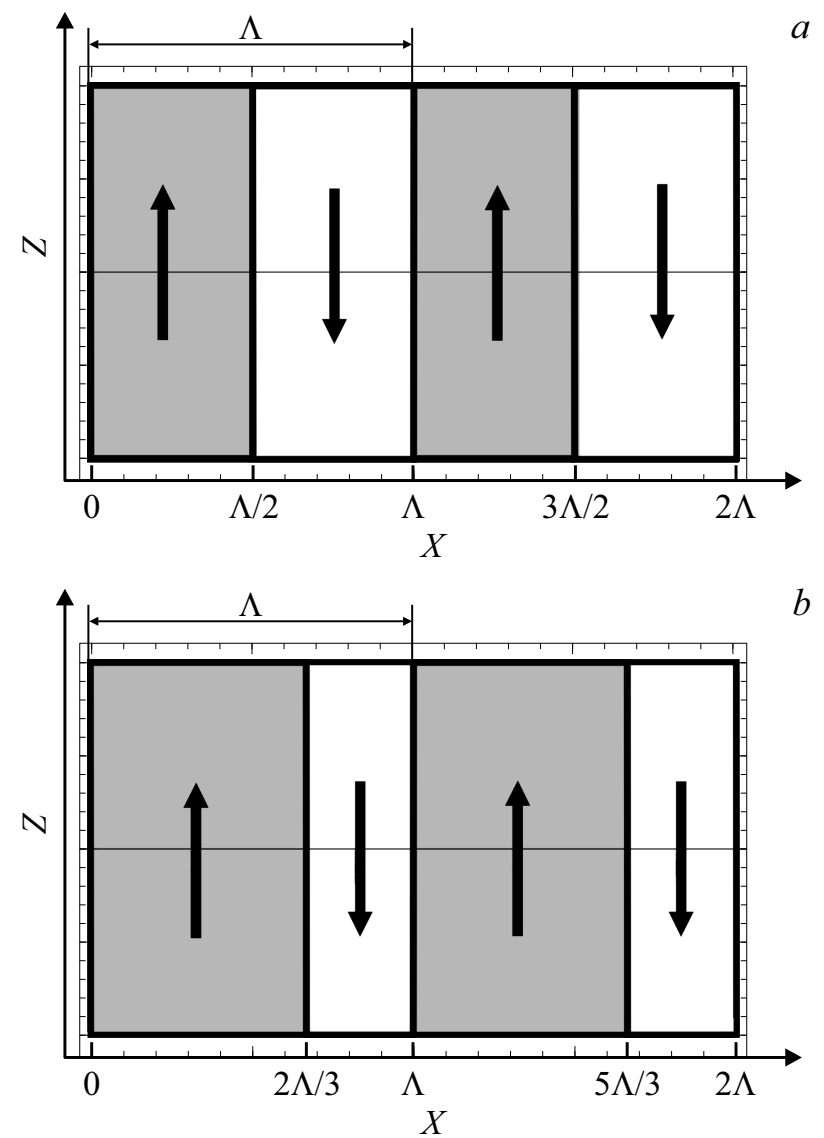

Рис. 2. Схематическое изображение модуляции нелинейного коэффициента $g(x)$ с периодом решетки $\Lambda$, за счет периодического ориентирования нелинейного кристалла, для симметричной $(a)$ (положительные и отрицательные домены имеют одинаковую длину) и несимметричной $(b)$ доменной структуры (положительные домены в 2 раза больше отрицательных). Направление периодической ориентации в домене отображается стрелками.

На рис. 3 приведены абсолютные значения коэффициентов Фурье функции $g(x)$ для случая симметричной $(a)$ и несимметричной $(b)$ решетки периодической ориентации. Из рис. 3 видно, что для данного каскадного нелинейного процесса не подходит обычная симметричная решетка, так как соседние фурье-компоненты для нее не могут быть отличны от нуля одновременно в отличие от фурье-компонент несимметричной решетки. Например, для нелинейного кристалла $\mathrm{LiNbO}_{3}$ полный синхронизм возможен для приведенной выше несимметричной решетки с периодом $-\Lambda_{0} \approx 9.76 \mu$ м и длиной волны $Ф Г-\lambda \approx 1570 \mathrm{~nm}$, которой соответствует $m=5$. Скважность несимметричной решетки в нашем случае равнялась $2 / 3$, так как для данной скважности максимально значение произведения соседних фурьекомпонент.

При условии, что полный фазовый синхронизм достигнут, в (8) из всех слагаемых суммы можно оставить только резонансные члены и переписать уравнения дви- жения в более простом виде

$$
\begin{gathered}
\frac{d \hat{b}_{M}(x)}{d x}=\kappa^{(4)} g_{m} E_{z}^{4}, \\
\frac{d \hat{a}_{M}(x)}{d x}=2 \kappa^{(2)} g_{m-1}^{*} \hat{b}_{M}(x) \hat{a}_{M}^{\dagger}(x) .
\end{gathered}
$$

Далее, будем считать поле ЧГ классическим, так же как и поле ФГ. Интегрируя первое уравнение в (11) при условии, что при $z=0$ поле ЧГ равно нулю, и подставляя значение $b_{M}(x)$ во второе уравнение, получим

$$
\begin{gathered}
b_{M}(x)=\beta x, \\
\frac{d \hat{a}_{M}(x)}{d x}=\alpha x \hat{a}_{M}^{\dagger}(x),
\end{gathered}
$$

где были введены обозначения $\alpha=2 E_{z}^{4} \kappa^{(2)} \kappa^{(4)} g_{m-1}^{*} g_{m}$ и $\beta=E_{z}^{4} \kappa^{(4)} g_{m}$, которые определяют эффективность процесса генерации ВГ и ЧГ соответственно. В результате поле ЧГ растет линейно внутри кристалла, а уравнение на ВГ свелось к операторному уравнению, которое почти совпадает с уравнением параметрического распада $[10]$, однако отличается от него наличием линейной функции по $x$ в правой части. В результате несложно найти решение такого модифицированного операторного уравнения, которое будет иметь вид

$$
\begin{gathered}
\hat{a}_{M}(x)=\operatorname{ch}\left(\frac{|\alpha| x^{2}}{2}\right) \hat{a}+e^{i \theta} \operatorname{sh}\left(\frac{|\alpha| x^{2}}{2}\right) \hat{a}^{\dagger}, \\
\hat{a}_{M}^{\dagger}(x)=\operatorname{ch}\left(\frac{|\alpha| x^{2}}{2}\right) \hat{a}+e^{-i \theta} \operatorname{sh}\left(\frac{|\alpha| x^{2}}{2}\right) \hat{a},
\end{gathered}
$$

где были использованы следующие операторные начальные условия: $a_{M}(x=0)=a, a_{M}^{\dagger}(x=0)=a^{\dagger}$, а за $\theta$ принимается аргумент комплексного числа $\alpha$. Предполагая, что начальное состояние для ВГ это вакуум, среднее число фотонов при распространении внутри кристалла будет расти по следующему закону:

$$
N_{2 \omega}(x)=\left\langle 0\left|\hat{a}_{M}^{\dagger}(x) \hat{a}_{M}(x)\right| 0\right\rangle=\operatorname{sh}^{2}\left(\frac{|\alpha| x^{2}}{2}\right) .
$$

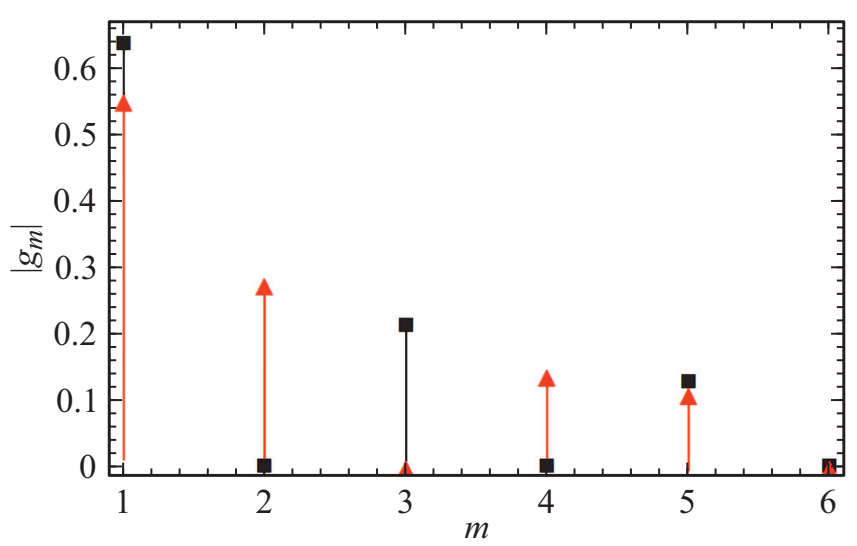

Рис. 3. Абсолютное значение коэффициентов Фурье функции $g(x)$ для симметричной (черные квадраты) и несимметричной решетки (красные треугольники) периодической ориентации кристалла. 


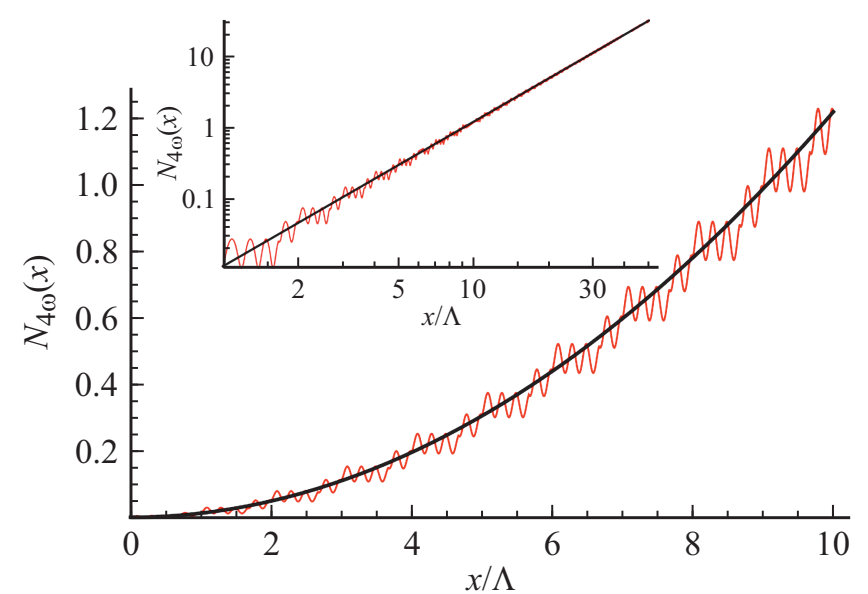

Рис. 4. Среднее число частиц четвертой гармоники, рассчитанное с помощью точной формулы (15) (красная тонкая кривая) и формулы (12) (черная толстая кривая), которая учитывает только резонансный вклад. На вставке показан график в двойном логарифмическом масштабе. Координата х нормируется на период решетки периодической поляризации $\Lambda$.

Таким образом, в отличие от обычного параметрического распада, для которого аргумент растет линейно как функция $x$, аргумент для рассмотренного каскадного процесса ГВГ будет расти квадратично по $x$.

Проверим насколько представленные решения для среднего числа частиц ВГ и ЧГ, полученные только при учете резонансных слагаемых из ряда фурье-функции $g(x)$ в (8), отличаются от точных решений, для которых необходимо учитывать явный вид функции $g(x)$. Точное решение для поле ЧГ можно получить, проинтегрировав уравнения (8), в приближении классического поля ЧГ и отсутствия влияния ВГ на поле ЧГ:

$$
b_{M}(x)=\kappa^{(4)} E_{z}^{4} \int_{0}^{x} d y g(y) e^{-i \Delta k y}
$$

На рис. 4 представлено сравнение среднего числа частиц ЧГ $N_{4 \omega}=b_{M}^{*} b_{M}$, рассчитанное с помощью формулы (12), которая учитывает только резонансный вклад, и формулы (15), которая содержит полную зависимость от функции $g(x)$. Из рис. 4 можно увидеть, что резонансное решение является огибающим для точного решения. Заметим, что функция $g(x)$ соответствует несимметричной решетке, показанной на рис. $2, b$.

Следующим шагом необходимо найти уравнение движения на среднее значение оператора числа частиц ВГ. Воспользовавшись (5), получим

$$
\begin{aligned}
\frac{d N_{2 \omega}(x)}{d x} & =\frac{d\left\langle\hat{a}_{M}^{\dagger}(x) \hat{a}_{M}(x)\right\rangle}{d x} \\
& =2 \kappa^{(2)} g(x) e^{-i(\Delta k-\delta k) x} b_{M}^{*}(x)\left\langle\hat{a}_{M}^{2}(x)\right\rangle+\text { h.c. },
\end{aligned}
$$

где h.c. обозначает эрмитово сопряженный оператор. В правой части уравнения (16) появляется среднее квадрата оператора уничтожения $\left\langle\hat{a}_{M}^{2}(x)\right\rangle$, уравнение движения для которого будет иметь вид

$$
\frac{d\left\langle\hat{a}_{M}^{2}(x)\right\rangle}{d x}=4 \kappa^{(2)} g(x) e^{i(\Delta k-\delta k) x} b_{M}(x)\left(\frac{1}{2}+N_{2 \omega}(x)\right) .
$$

Необходимо уточнить, что $1 / 2$ в правой части уравнения (17) появляется за счет квантовых флуктуаций и отсутствует при классическом рассмотрении данного нелинейного процесса, а стоящее правее среднее число частиц приводит к вынужденной генерации для $N_{2 \omega} \gg 1$. Таким образом, система из уравнений (16) и (17) на среднее число частиц ВГ является замкнутой, что позволяет решить ее численными методами. На рис. 5 изображено сравнение численно рассчитанной зависимости среднего числа частиц ВГ от координаты $x$ и огибающей, которая была получена из учета только резонансного слагаемого. Загиб кривой на рис. 5 для графика в логарифмическом масштабе на больших длинах объясняется переходом генерации в упомянутый выше вынужденный режим. В этом случае число фотонов ВГ начинает расти по экспоненциальному закону вместо степенного.

Выше рассматривалась ситуация, для которой фазовый синхронизм был выполнен как для ГВГ, так и для ЧГ. Как известно, в каскадных процессах необязательно наличие фазового синхронизма для промежуточной частицы, роль которой в рассматриваемом нелинейном процессе играет фотон ЧГ [11] и в общем случае достаточно выполнения условия только для фазового синхронизма ГВГ (10). Чтобы понять, каким образом изменятся уравнения, описывающие процесс ГВГ, вернемся к формуле (8). Выразив в явном виде

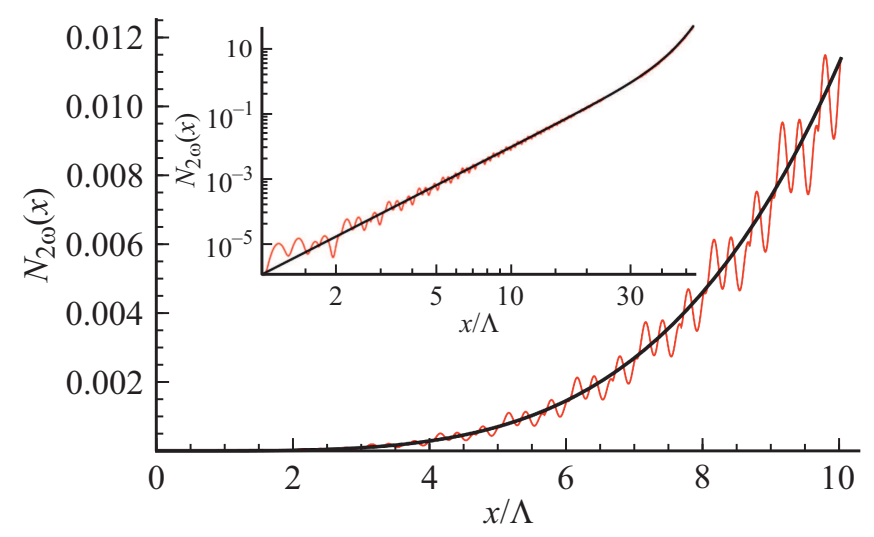

Рис. 5. Среднее число частиц ВГ, рассчитанное с помощью точной системы уравнений (16) и (красная тонкая кривая) и формулы (14) (черная толстая кривая), которая учитывает только резонансный вклад. На вставке показан график в двойном логарифмическом масштабе. Отклонение от линейного роста для больших $x$ связанно с экспоненциальным ростом числа частиц второй гармоники. Координата $x$ нормируется на период решетки периодической поляризации $\Lambda$. 
функцию „ $b_{M}$ “, подставив ее во второе уравнение (8) и воспользовавшись условием (10) для случая ГВГ с фазовым синхронизмом половинного порядка, получим

$$
\frac{d \hat{a}_{M}(x)}{d x}=\alpha\left[\frac{i\left(1-e^{i \Delta_{m} x}\right)}{\Delta_{m}}\right] \hat{a}_{M}^{\dagger}(x),
$$

где мы снова учли только одно резонансное слагаемое из всего фурье-разложения функции $g(x)$ и для краткости ввели обозначение $\Delta_{m}=\Delta k-m q_{0}$. Если в выражении в квадратных скобках устремить $\Delta_{m} \rightarrow 0$, что соответствует условию полного фазового синхронизма, то мы снова получим второе уравнение из (12). Если считать $\Delta_{m}$ конечной величиной, то в пространственной области, где $x \gg 2 \pi / \Delta_{m}$, главный вклад в квадратной скобке выражения (18) будет давать независящее от $x$ слагаемое, что приведет к перестройке темпа ГВГ с квадратично растущей функции $x$ на линейную вблизи точки $x \approx 2 \pi / \Delta_{m}$. Таким образом, количество частиц в области $x \gg 2 \pi / \Delta_{m}$ будет определяться следующей формулой:

$$
N_{2 \omega}(x)=\left\langle 0\left|\hat{a}_{M}^{\dagger}(x) \hat{a}_{M}(x)\right| 0\right\rangle=\operatorname{sh}^{2}\left(|\alpha| x / \Delta_{m}\right) .
$$

В результате было показано, что для данного нелинейного процесса при неполном промежуточном фазовом синхронизме ГВГ также осуществима.

\section{Заключение}

Таким образом, показано, что нелинейный каскадный процесс, в котором две пары фотонов фундаментальной гармоники преобразовываются в два фотона второй гармоники через промежуточный фотон четвертой гармоники, обеспечивает генерацию при дробном порядке периодической ориентации нелинейного кристалла. Предсказано, что половинный порядок фазового синхронизма будет наблюдаться для случая несимметричной решетки периодической ориентации. Исследованы режимы генерации как для полного фазового синхронизма всех нелинейных процессов, так и для частичного синхронизма, в котором отсутствует фазовый синхронизм для промежуточного процесса генерации четвертой гармоники. В дальнейшем планируется изучить влияние волноводных эффектов [12], а также различных типов решетки периодической ориентации [13].

\section{Конфликт интересов}

Авторы заявляют, что у них нет конфликта интересов.

\section{Список литературы}

[1] Fedorova K.A., Sokolovskii G.S., Nikitichev D.I., Battle P.R., Krestnikov I.L., Livshits D.A., Rafailov E.U. // Opt. Lett. 2013. V. 38. N 15. P. 2835.

[2] Rein B., Heck J., Walther T. // Laser Phys. Lett. 2017. V. 14. N 9. P. 095001.
[3] Christensen M., Hansen A.K., Noordegraaf D., Jensen O.B., Skovgaard P.M.W. // Appl. Opt. 2017. V. 56. N 8. P. 2250.

[4] Armstrong J.A., Bloembergen N., Ducuing J., Pershan P.S. // Phys. Rev. 1962. V. 127. N 6. P. 1918-1939.

[5] Fejer M.M., Jundt D.H., Byer R.L., Magel G.A. // IEEE J. Quantum Electron. 1992. V. 28. N 11. P. 2631-2654.

[6] Dudelev V.V., Fedorova K.A., Chistyakov D.V., Soboleva K.K., Bugrov V.E., Rafailov E.U., Sokolovskii G.S. // Proc. International Conference Laser Optics 2018. ICLO, 2018. P. 175.

[7] Sokolovskaya E.G., Fedorova K.A., Dudelev V.V., Rafailov E.U., Sokolovskii G.S. // J. Phys. Conf. Ser. 2016. P. 769. N 1. P. 012050. doi 10.1088/1742-6596/769/1/012050

[8] Huttner B., Serulnik S., Ben-Aryeh Y. // Phys. Rev. A. 1990. V. 42. N 9. P. 5594-5600.

[9] Caves C.M., Crouch D.D. // J. Opt. Soc. Am. B. 1987. V. 4. N 10. P. 1535.

[10] Mandel L., Wolf E. // Optical Coherence and Quantum Optics. Cambridge University, 1995. P. 1076.

[11] Klyshko D.N. Photons And Nonlinear Optics. Glasgow: CRC Press, 1988. P. 313.

[12] Chestnut D.A., Popov S.V., Taylor J.R., Roberts T.D. // Appl. Phys. Lett. 2006. V. 88. N 7. P. 071113.

[13] Bahabad A., Voloch N., Arie A., Lifshitz R. // J. Opt. Soc. Am. B. 2007. V. 24. N 8. P. 1916. 